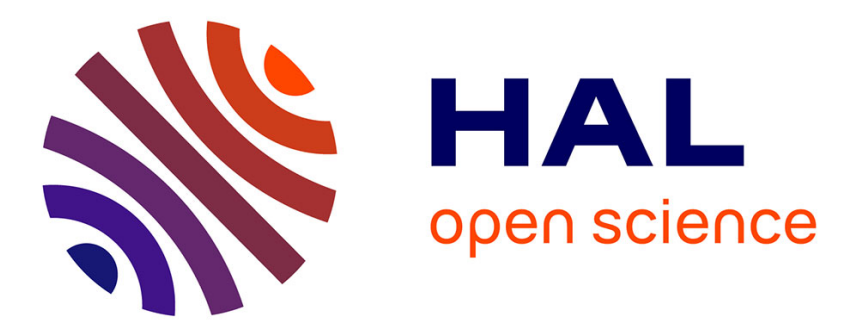

\title{
Rheological modeling of carbon nanotube aggregate suspensions
}

\author{
Anson Ma, Francisco Chinesta, Malcom Mackley
}

\section{To cite this version:}

Anson Ma, Francisco Chinesta, Malcom Mackley. Rheological modeling of carbon nanotube aggregate suspensions. Journal of Rheology, 2009, 52 (6), pp.1311 - 1330. 10.1122/1.2982932 . hal-01007375

\section{HAL Id: hal-01007375 \\ https://hal.science/hal-01007375}

Submitted on 15 Apr 2017

HAL is a multi-disciplinary open access archive for the deposit and dissemination of scientific research documents, whether they are published or not. The documents may come from teaching and research institutions in France or abroad, or from public or private research centers.
L'archive ouverte pluridisciplinaire HAL, est destinée au dépôt et à la diffusion de documents scientifiques de niveau recherche, publiés ou non, émanant des établissements d'enseignement et de recherche français ou étrangers, des laboratoires publics ou privés. 


\title{
Rheological modeling of carbon nanotube aggregate suspensions
}

\author{
W. K. A. Ma \\ Department of Chemical Engineering, University of Cambridge, Pembroke Street, \\ Cambridge CB2 3RA, United Kingdom \\ F. Chinesta \\ Laboratoire de Mécanique des Systèmes et des Procédés, \\ 151 Boulevard de l'Hôpital, 75013, Paris, France
}

\begin{abstract}
A. Ammar
Laboratoire de Rhéologie, Domaine Universitaire, 1301 Rue de la piscine, 38041 Grenoble Cedex 9, France
\end{abstract}

\author{
M. R. Mackley \\ Department of Chemical Engineering, University of Cambridge, Pembroke Street, \\ Cambridge CB2 3RA, United Kingdom
}

This paper is concerned with the rheological modeling of untreated carbon nanotubes (CNTs) suspended within an epoxy resin. The untreated CNT suspensions exhibited significant steady shear thinning and contained optically resolvable aggregate structures. A simple orientation model, based on a Fokker-Planck advection-diffusion description, failed to capture the experimentally observed rheological responses for untreated CNT suspensions. A new model named the "aggregation/orientation" (AO) model has been developed to describe the experimental findings. The model integrated elements of both a standard orientation model and aggregation modeling concepts within the Fokker-Planck formalism. A hierarchy of states between CNTs that are free from entanglement and a complete CNT network was incorporated into the AO model, thereby enabling different microstructure populations to exist for different shear conditions. Using a small number of adjustable parameters, it was found that the experimental data could be fitted with reasonable precision.

\section{INTRODUCTION}

The experimental rheology of carbon nanotubes (CNTs) has been studied extensively over the last few years [see, for example, Kinloch et al. (2002); McNally et al. (2005); 
Rahatekar et al. (2006)] and the rheological behavior of treated and untreated CNT suspensions has been found to be significantly different particularly in relation to shear thinning. Several authors [see, for example, Song and Youn (2005); Fan and Advani (2007)] reported mild shear thinning and viscoelasticity for treated CNT suspensions, whereas Rahatekar et al. (2006) showed that untreated CNT suspensions exhibited significant steady shear-thinning characteristics. In addition, a number of authors reported that there was a significant dependence of mixing protocols and time, in relation to the rheological response of CNT suspensions [Huang et al. (2006); Fan and Advani (2007)]. There are a number of publications on linear viscoelasticity (LVE) [see, for example, Pötschke et al. (2002); Du et al. (2004); Abdel-Goad and Pötschke (2005)] and also modeling of both treated and untreated CNT suspensions [Hough et al. (2004); Xu et al. (2005)].

At present there is no universal consensus to describe experimental LVE data for surfactant stablized or treated CNT suspensions. Hough and co-workers (2004) suggested that the experimentally observed elasticity was due to interaction between CNTs in a network, whilst Xu et al. (2005) implied in their Maxwell-type modeling that elasticity possibly resulted from stretching and bending of CNTs. In the case of aggregating CNT suspensions, Hobbie and Fry (2007) measured the shear thinning and LVE responses of semidilute to concentrated CNT suspensions and related these responses to the morphology of the CNT network.

In terms of direct visualization, there are publications on the aggregates or bundles of CNTs [Lin-Gibson et al. (2004); Hobbie and Fry (2006); Rahatekar et al. (2006); Ma et al. (2007)] and subsequently mapping different CNT aggregate structures into a phase diagram [Davis et al. (2004); Hobbie and Fry (2006)]. Fry et al. (2005) measured the anisotropy of sheared CNT suspensions using small-angle neutron scattering and smallangle light scattering techniques. In general, optical microstructures were observed in untreated CNTs, whereas suspensions with treated CNTs tended to show less optical texture [Xu et al. (2005); Ma et al. (2008a)], making structural characterization of treated CNT suspensions difficult. Nanoscale characterization is possible using techniques such as atomic force microscopy, scanning electron microscopy, and transmission electron microscopy; however, these techniques work best with solid samples and usually require thermo/photocuring or cryogenic treatments. Fan and Advani (2005, 2007), for example, showed electron micrographs of CNTs that were preferentially aligned in the flow direction after applying shear to the suspension, followed by subsequent thermocuring of the epoxy matrix. More recently, Ma et al. (2008b) reported the photocuring of CNT suspensions within a rheometer as a means to preserve shear-induced microstructures.

In terms of modeling, the evolution of flow curves for CNT suspensions can be described by different empirical models such as the Cross model, the Carreau model, and the Krieger-Dougherty model [Kharchenko et al. (2004); Rahatekar et al. (2006); Kim et al. (2005)]. A stress-microstructure model, however, is desirable as information such as CNT orientation and state of aggregation is intimately related to the final physical properties of CNT composite materials [Song and Youn (2005)]. The current CNT modeling is based on both fibre orientation models [Jeffery (1922); Batchelor (1970); Hinch and Leal (1975, 1976); Petrich et al. (2000)] and transient network modeling concepts [Vaccaro and Marrucci (2000); Hernandez Cifre et al. (2003); Rincón et al. (2005)]. In the case of flocculating fiber suspensions, Klingenberg and co-workers performed particlelevel dynamic simulations considering fiber flexibility and fiber interactions [Schmid et al. (2000); Schmid and Klingenberg (2000); Switzer and Klingenberg (2004)], whilst Chaouche and Koch (2001) further associated shear thinning of fiber suspensions to the formation and breakage of fiber flocs. Orientation models have been reviewed in a num- 
ber of publications [Zirnsak et al. (1994); Larson (1999); Petrie (1999)] and in terms of transient network modeling, the paper of Tripathi et al. (2006) summarized different types of transient-network construction/destruction kinetics reported in the literature.

The first part of the paper describes experimental results for shear thinning and optical textures. It is then shown that a simple Fokker-Planck based orientation model is inadequate at explaining the observed experimental results and therefore a subsequent more advanced model has been developed. The new model incorporates both the elements of orientation modeling and aggregation modeling concepts. Finally, the new model is tested against the experimental data and the effect of different adjustable parameters is explored.

\section{EXPERIMENT}

In this study, the CNTs were supplied by the Department of Materials Science and Metallurgy at the University of Cambridge. They were multiwalled CNTs produced by the chemical vapor deposition method [Singh et al. (2003)] and no chemical treatment has been applied to the CNTs reported in this paper. Although the diameter and length of the CNTs have not been fully characterized, SEM indicated that individual CNT had a typical diameter of less than $100 \mathrm{~nm}$ and optical microscopy suggested a typical length of about $30 \mu \mathrm{m}$. The CNTs have an aspect ratio $(r)$ of about 300 and according to Petrie (1999), the transition from semidilute to concentrated regime for randomly orientated fibers occurs at $1 / r \approx 0.3 \%$. The CNTs were dispersed in an epoxy resin (Araldiate LY556, Huntsman, Inc.) and a high-shear homogenizer (Silverson L4R) was used for the preparation of a masterbatch suspension containing $0.25 \%$ CNT (by weight). Lower concentration samples were prepared from diluting the masterbatch. A mixing time of $5 \mathrm{~h}$ was used to ensure the establishment of stable rheology [Huang et al. (2006)].

Rheological measurements were carried out using an ARES strain-controlled rheometer (TA Instruments) with $50 \mathrm{~mm}$ parallel plate, a gap size of $500 \mu \mathrm{m}$, and at a temperature of $25^{\circ} \mathrm{C}$. Steady shear viscosity was measured after a shearing time of $100 \mathrm{~s}$ and shear gradient changes within the parallel-plate rheometry was corrected using the method described by Macosko (1994). The correction method has been widely used and was suggested to be applicable to power-law fluids $\left(\tau=K \dot{\gamma}^{n}\right)$ with $n<1.2$ [Carvalho et al. (1994)]. Optical microstructure studies were carried out using a Cambridge Shear System (CSS 450, manufactured by Linkam Scientific Instruments), which consists of a shear cell coupled with an optical microscope. The shear cell is composed of two parallel quartz disks, where the bottom disk can rotate and the rotation speed is controlled by a stepper motor. The system allowed direct observation of optical microstructure developed within the CNT suspension for different shear conditions. Optical observations were made at a position $7.5 \mathrm{~mm}$ from the center of $30 \mathrm{~mm}$ quartz disks and all reported shear rates were referenced to this position. Optical images were captured using a JVC color video camera (TK-C1480E) with a frame rate of $1 / 1000 \mathrm{~s}$ and optical textures seen during and immediately after shear were essentially the same.

When dispersed in an epoxy resin, both treated and untreated CNTs showed steady shear-thinning characteristics, but to a different extent (Fig. 1). The epoxy matrix is optically transparent, essentially Newtonian and with a base viscosity of $10 \mathrm{~Pa}$ s. As shown in the figure, untreated CNT suspensions possessed a higher apparent low-shear viscosity coupled with optical microstructure of CNT aggregates, whereas treated CNT suspensions showed no optically resolvable CNT aggregates with a less pronounced viscosity enhancement effect.

The steady shear rheology of untreated CNT suspensions was studied in greater detail and the evolution of apparent shear viscosity $\left(\eta_{a}\right)$ as a function of shear rate is shown in 


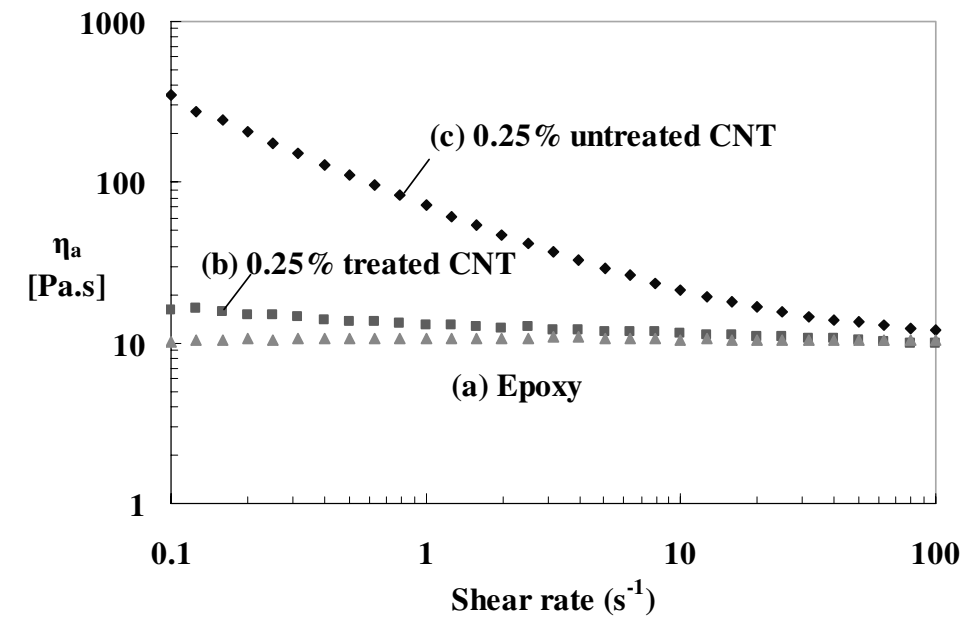

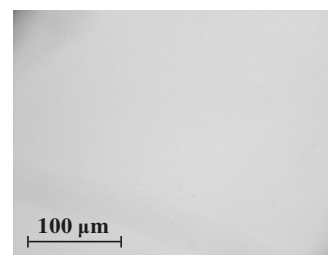

(a) Epoxy

$\left(\right.$ Shear rate $\left.=0 \mathrm{~s}^{-1}\right)$

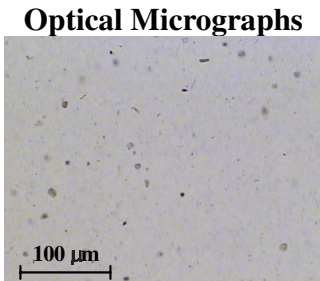

(b) $0.25 \%$ treated CNT $\left(\right.$ Shear rate $\left.=0 \mathrm{~s}^{-1}\right)$

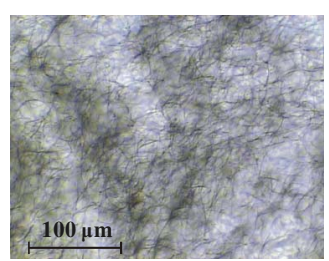

(c) $0.25 \%$ untreated CNT $\left(\right.$ Shear rate $\left.=0 \mathrm{~s}^{-1}\right)$

FIG. 1. Apparent shear viscosity $\left(\eta_{a}\right)$ of (a) epoxy, (b) $0.25 \%$ treated CNT, and (c) $0.25 \%$ untreated CNT suspensions. Treated and untreated CNTs both exhibited shear-thinning characteristics but to a different extent when dispersed in an epoxy matrix. Untreated CNTs showed a higher low-shear viscosity coupled with optical microstructures of CNT aggregates whereas treated CNTs showed no clear optically resolvable CNT aggregates with a less pronounced viscosity enhancement effect. (Treated CNTs were supplied by Nanocomposites Inc., USA, and the same mixing protocol was used for preparing both treated and untreated CNT suspensions.)

Fig. 2 for four different concentrations. The untreated CNT suspensions exhibited a significant viscosity enhancement at low shear rates and it was observed that the addition of only $0.1 \%$ CNT had led to an order-of-magnitude increase in $\eta_{a}$. At high shear rates, the viscosity of the suspensions decreased asymptotically to that of the base epoxy and these findings are consistent with those reported by Rahatekar et al. (2006). In terms of microstructure, untreated CNT suspensions showed a clear optical texture which evolved depending on the shear conditions [see for example Figs. 3(a)-3(d)]. At low shear rates, coarsening of the optical texture was generally observed after applying steady shear for a certain period of time [as shown in Fig. 3(b)]. At high shear rates, CNT suspensions were more uniformly dispersed and the aggregate size of CNTs decreased as shear rate increased [Figs. 3(c) and 3(d)].

\section{FOKKER-PLANCK ORIENTATION MODEL}

In the context of short-fiber suspensions modeling, an orientation model can be used to describe the evolution of steady shear viscosity by coupling the flow kinematics with fiber orientation [see, for example, Batchelor (1970); Hand (1962); Hinch and Leal (1975, 1976); review by Petrie (1999)]. Initially, a Fokker-Planck (FP) model was as- 


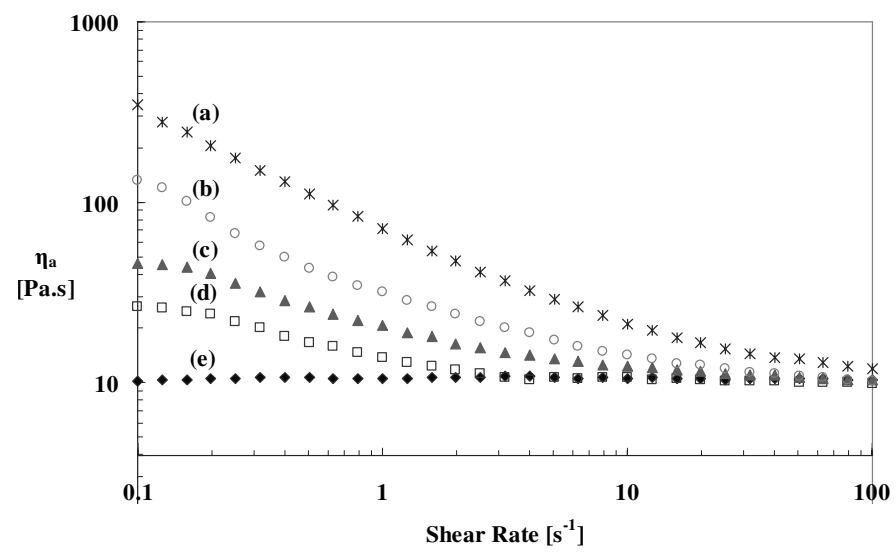

FIG. 2. Apparent shear viscosity $\left(\eta_{a}\right)$ as a function of shear rate for (a) $0.25 \% \mathrm{CNT}$, (b) $0.1 \%$, (c) $0.05 \%$, (d) $0.025 \%$ CNT suspensions, and (e) epoxy only. The $\eta_{a}$ data presented are experimental data and the values were obtained after a shearing time of $100 \mathrm{~s}$.

sumed where the CNTs were considered as short, rigid fibers that can rotate and align in a shear flow and where the evolution of the viscosity contribution due to the presence of CNTs depended only on the orientation of CNTs.

\section{A. Formulation of the FP model}

The momentum balance equation (neglecting the inertia and mass terms)

$$
\operatorname{Div} \boldsymbol{\sigma}=\mathbf{0},
$$

where $\boldsymbol{\sigma}$ is the stress tensor.

The mass balance equation for an incompressible fluid is given as

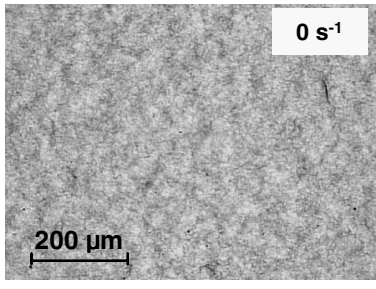

(a)

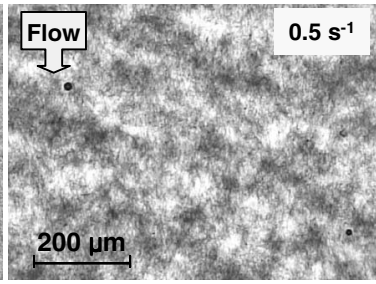

(c)

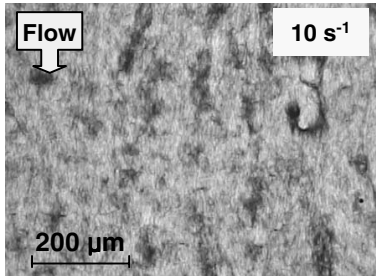

(b)

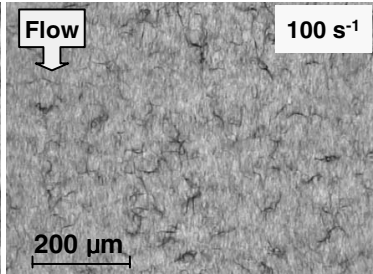

(d)

FIG. 3. Optical microstructure of $0.1 \%$ CNT suspended within an epoxy resin after shearing at (a) 0 , (b) 0.5 , (c) 10 , and (d) $100 \mathrm{~s}^{-1}$. The micrographs were captured after a shearing time of $100 \mathrm{~s}$ and using the Cambridge Shear System (CSS 450) with an optical depth of $130 \mu \mathrm{m}$. 
where $\mathbf{v}$ represents the velocity field.

The constitutive equation for a dilute suspension of high aspect-ratio particles (with negligible Brownian diffusion) can be written as

$$
\boldsymbol{\sigma}=-p \mathbf{I}+2 \eta\left\{\mathbf{D}+N_{p}\left(\mathbf{a}_{4}: \mathbf{D}\right)\right\}
$$

where $p$ denotes the hydrostatic pressure, $\mathbf{I}$ is the unit tensor, $\eta$ is the matrix viscosity, $\mathbf{D}$ is the strain-rate tensor, $N_{p}$ is a scalar parameter that depends on fiber concentration and aspect ratio, ":" is the tensor product twice contracted [i.e., $\left.\left(\mathbf{a}_{4}: \mathbf{D}\right)_{i j}=\left(\mathbf{a}_{4}\right)_{i j k l} \mathbf{D}_{k l}\right]$ and $\mathbf{a}_{4}$ is the fourth order orientation tensor and it is defined as

$$
\mathbf{a}_{4}=\int \boldsymbol{\rho} \otimes \boldsymbol{\rho} \otimes \boldsymbol{\rho} \otimes \boldsymbol{\rho} \psi(\boldsymbol{\rho}) d \boldsymbol{\rho},
$$

where $\boldsymbol{\rho}$ is the unit vector aligned in the fiber axis direction, " $\otimes$ " denotes the tensorial product [i.e., $\left.(\boldsymbol{\rho} \otimes \boldsymbol{\rho})_{i j}=\boldsymbol{\rho}_{i} \boldsymbol{\rho}_{j}\right]$, and $\psi(\boldsymbol{\rho})$ is the orientation distribution function which satisfies the normality condition $\int \psi(\boldsymbol{\rho}) d \boldsymbol{\rho}=1$. In general, the distribution function depends on the physical coordinates $\mathbf{x}$, the time $t$, and the conformation coordinates $\boldsymbol{\rho}$ [i.e., $\psi(\mathbf{x}, t, \boldsymbol{\rho})]$, but if the flow is homogeneous and steady, the fiber orientation distribution would only depend on the conformation coordinates [i.e., $\psi(\boldsymbol{\rho})]$. For dilute and nonaggregating short fiber suspensions, different analytical expressions for the parameter $N_{p}$ are available [Lipscomb et al. (1988); Petrie (1999)]. However, those expressions do not immediately apply to semidilute/concentrated or aggregating suspensions. Aggregation has been observed within the semidilute untreated CNT suspensions studied in this paper and $N_{p}$ is therefore determined from experimental data fitting.

If all the fibers are completely aligned in the direction $\hat{\boldsymbol{\rho}}[$ i.e., $\psi(\boldsymbol{\rho})=\delta(\boldsymbol{\rho}-\hat{\boldsymbol{\rho}})$, where $\delta()$ is the Dirac's distribution], then the corresponding fourth-order orientation tensor would become: $\mathbf{a}_{4}=\hat{\boldsymbol{\rho}} \otimes \hat{\boldsymbol{\rho}} \otimes \hat{\boldsymbol{\rho}} \otimes \hat{\boldsymbol{\rho}}$ and the fourth-order orientation tensor can be written in the following form:

$$
\mathbf{a}_{4}=\mathbf{a}_{2} \otimes \mathbf{a}_{2}
$$

where $\mathbf{a}_{2}=\int \boldsymbol{\rho} \otimes \boldsymbol{\rho} \psi(\boldsymbol{\rho}) d \boldsymbol{\rho}$ denotes the second order orientation tensor and corresponding components are given as $\left(\mathbf{a}_{4}\right)_{i j k l}=\left(\mathbf{a}_{2}\right)_{i j}\left(\mathbf{a}_{2}\right)_{k l}$.

In cases where the fibers are not completely aligned in one direction, Eq. (5) is not exact, but it provides an approximation for the fourth-order orientation tensor. Equation (5) is also known as the quadratic closure relation: $\mathbf{a}_{4}^{\text {quad }}=\mathbf{a}_{2} \otimes \mathbf{a}_{2}$ and other forms of closure relations are also available in the literature [see, for example, Advani and Tucker (1990); Dupret et al. (1998)]. However, it is also generally accepted that these closure approximations are not desirable for computing the exact orientation tensor because their impact on the final fiber distribution can sometimes be very large.

The orientation distribution function $\psi(\boldsymbol{\rho})$ can be determined by solving its balance equation, which is also known as the FP equation

$$
\frac{D \psi(\boldsymbol{\rho})}{D t}+\frac{\partial}{\partial \boldsymbol{\rho}} \cdot\left(\psi(\boldsymbol{\rho}) \frac{d \boldsymbol{\rho}}{d t}\right)=0,
$$

where $D \psi / D t$ is the material derivative and $D \psi / D t=\partial \psi / \partial t+(\mathbf{v} \cdot \nabla) \psi$. Once the distribution function is calculated, the computation of its moments (orientation tensors) then becomes straightforward and does not require any closure approximation.

For a dilute suspension of spheroids and in the absence of Brownian motion, the evolution of fiber orientation was described by Jeffery (1922), 


$$
\frac{d \boldsymbol{\rho}}{d t}=\boldsymbol{\Omega} \cdot \boldsymbol{\rho}+k\left(\mathbf{D} \cdot \boldsymbol{\rho}-\left(\boldsymbol{\rho}^{T} \cdot \mathbf{D} \cdot \boldsymbol{\rho}\right) \boldsymbol{\rho}\right),
$$

where $\boldsymbol{\Omega}$ is the vorticity tensor and $k$ is a constant that depends on the fiber aspect ratio $r$ ( $r$ =fiber length/fiber diameter) and $k$ is given as

$$
k=\left(r^{2}-1\right) /\left(r^{2}+1\right) .
$$

For particles with an infinite aspect ratio $k \approx 1$, Eq. (7) reduces to

$$
\frac{d \boldsymbol{\rho}}{d t}=\boldsymbol{\nabla} \mathbf{v} \cdot \boldsymbol{\rho}-\left(\boldsymbol{\rho}^{T} \cdot \mathbf{D} \cdot \boldsymbol{\rho}\right) \boldsymbol{\rho} .
$$

By combining Eqs. (6) and (7), the equation governing the evolution of the second order orientation tensor can be deduced

$$
\frac{d \mathbf{a}_{2}}{d t}=\mathbf{\Omega} \cdot \mathbf{a}_{2}-\mathbf{a}_{2} \cdot \mathbf{\Omega}+k\left(\mathbf{D} \cdot \mathbf{a}_{2}+\mathbf{a}_{2} \cdot \mathbf{D}-2\left(\mathbf{a}_{4}: \mathbf{D}\right)\right)
$$

In the case of semidilute fiber suspensions, Folgar and Tucker (1984) proposed the introduction of a diffusion term $\left(D_{r}\right)$ in Eq. (6) to account for fiber interaction effects, resulting in the following expression:

$$
\frac{d \psi(\boldsymbol{\rho})}{d t}+\frac{\partial}{\partial \boldsymbol{\rho}} \cdot\left\{\psi(\boldsymbol{\rho}) \frac{d \boldsymbol{\rho}}{d t}\right\}=\frac{\partial}{\partial \boldsymbol{\rho}} \cdot\left\{D_{r} \frac{\partial \psi(\boldsymbol{\rho})}{\partial \boldsymbol{\rho}}\right\} .
$$

The equation governing the evolution of $\mathbf{a}_{2}$ [in three dimensions (3D)] then becomes

$$
\frac{d \mathbf{a}_{2}}{d t}=\mathbf{\Omega} \cdot \mathbf{a}_{2}-\mathbf{a}_{2} \cdot \boldsymbol{\Omega}+k\left(\mathbf{D} \cdot \mathbf{a}_{2}+\mathbf{a}_{2} \cdot \mathbf{D}-2\left(\mathbf{a}_{4}: \mathbf{D}\right)\right)-6 D_{r}\left(\mathbf{a}_{2}-\frac{\mathbf{I}}{3}\right) .
$$

Equation (12) shows that the evolution equation for the second-order orientation tensor involves the fourth-order orientation tensor and in order to solve the problem in a closed form, closure approximations which express the fourth-order orientation tensor in terms of lower-order tensors are commonly used [Advani and Tucker (1990); Dupret and Verleye (1999)]. Instead of using closure approximations, a number of authors solved the FP description directly [see, for example, Chinesta et al. (2003); Keunings (2004); Ammar et al. (2006)]. However, the latter approach also requires the computation of a multidimensional distribution function and thus involves considerable computation efforts. In this study, the FP equation has been solved directly to allow for an accurate evaluation of the orientation tensor.

\section{B. Application of the FP model}

In the FP based orientation model, $N_{p}$ and $D_{r}$ are the key adjustable parameters to fit with experimental data. The model was found to be successful in capturing the shear thinning characteristic for some surface-treated CNT suspensions [Ma et al. (submitted)], where no optically resolvable CNT aggregate was observed. In the FP orientation modeling, the value of $N_{p}$ was adjusted to fit the initial evolution of viscosity at low shear rates and different values of $N_{p}$ were needed to describe the experimental data at different treated CNT concentration levels. At high shear rates, the apparent shear viscosity was fitted by adjusting the value of rotary diffusion coefficient $\left(D_{r}\right)$, which physically represents the rate of misalignment from the flow direction due to randomising events such as Brownian motion and tube-tube interactions. For a certain surface-treated CNT suspension (with a fixed $N_{p}$ ), a single $D_{r}$ value was found to be sufficient in describing the 
(a) $0.25 \%$ CNT

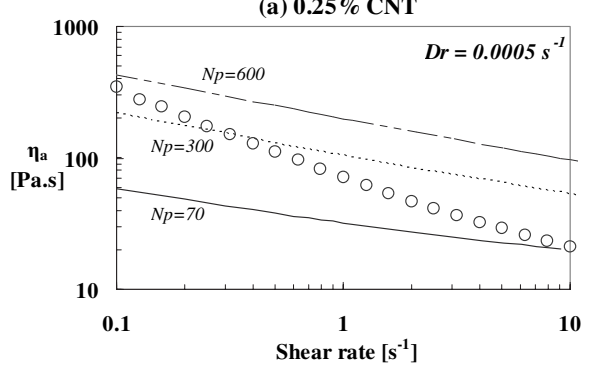

(c) $0.25 \% \mathrm{CNT}$

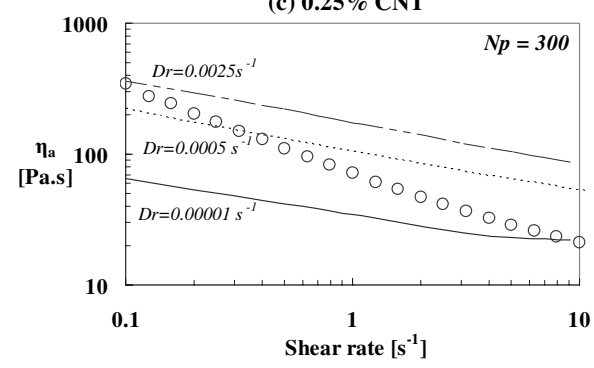

(b) $0.1 \% \mathrm{CNT}$

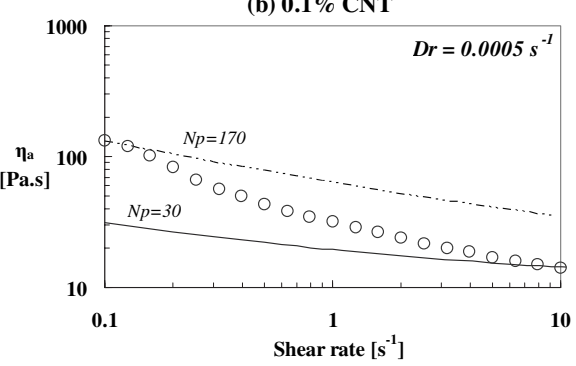

(d) $0.1 \%$ CNT

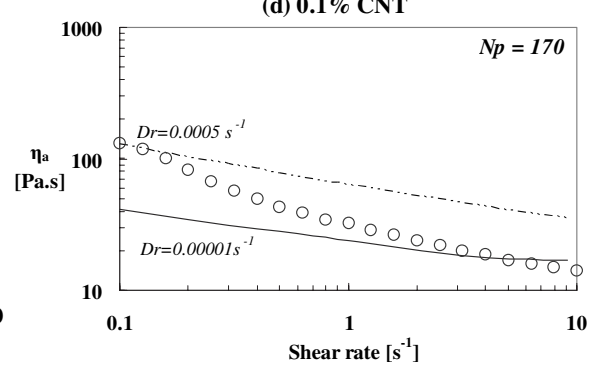

FIG. 4. FP orientation model fitting to experimental $\eta_{a}$ data at two selected CNT concentration levels where strong viscosity enhancement effect was observed [concentration $=0.25 \%$ for (a) and (c) and concentration $=0.1 \%$ for (b) and (d)]. (a) and (b) showed the fittings with a fixed rotary diffusion coefficient $\left(D_{r}\right.$ $=0.0005 \mathrm{~s}^{-1}$ ) and a changing $N_{p}$, whereas (c) and (d) showed the fittings with a fixed $N_{p}$, but with an adjustable $D_{r}$. Experimental data are represented by unfilled circles $(\bigcirc)$ and the lines are the FP model fits.

evolution of steady shear viscosity for a wide range of shear rates [Ma et al. (submitted)]. The success of this model for treated CNTs can be understood in terms of the nonaggregating characteristic of treated CNT suspensions, where CNT orientation was expected to be the key factor in controlling the evolution of steady shear viscosity.

The FP based orientation model with the use of a single $D_{r}$ and $N_{p}$ values, however, failed to fit the experimental flow curves for untreated CNT suspensions, where the viscosity enhancement was very pronounced at low shear rates. Figures 4(a) and 4(b) show FP orientation model fitting to experimental data at two selected concentrations using different values of $N_{p}$ and an arbitrarily fixed $D_{r}$, whereas in Figs. 4(c) and 4(d), the value of $D_{r}$ was adjusted (with $N_{p}$ fixed) in an attempt to fit the experimental data. The level of the fitting curve is more sensitive to the absolute value of $N_{p}$ rather than $D_{r}$. It is also clear from all these fittings [Figs. 4(a)-4(d)] that considering only CNT orientation was not sufficient in describing the observed viscosity enhancement. It is believed that the extra contribution to shear viscosity at low shear rates is due to the presence of CNT aggregates, which were experimentally observed in optical microstructure studies [see also Figs. 3(b)-3(d)].

\section{AGGREGATION/ORIENTATION MODEL}

\section{A. Formulation of the aggregation/orientation model for steady and homogeneous flows}

The FP orientation model was unable to describe the shear-thinning characteristic for untreated CNT suspensions, and therefore, a new model named the aggregation/ orientation (AO) model was developed. The new model considered a hierarchy of CNT aggregate structures within an untreated CNT suspension, where the shear viscosity was 
controlled by the state of aggregation and CNT orientation. The FP description was modified to incorporate aggregation/disaggregation kinetics and a detailed derivation for the AO model is included in this section.

The AO distribution function in the AO model is written as $\psi(\mathbf{x}, t, \boldsymbol{\rho}, n)$, where $n$ $\in[0,1]$ describes the state of aggregation ( $n=0$ corresponds to CNTs that are free from entanglement and $n=1$ represents a CNT aggregate network). It is assumed that the flow is steady and homogeneous and that different populations $(n)$ are present within the control volume considered in the balance equation. In more complex flow situations where spatial flow inhomogeneity is present, the material derivative in the FP equation contains the orientation distribution function gradient and it does not reduce to the temporal partial derivative. Such spatial dependence does not introduce great difficulties in numerical modeling and is commonly encountered in the simulation of forming processes involving flows in complex geometries. There is, however, also evidence in Fig. 3 showing local inhomogeneity in terms of CNT concentration. This type of inhomogeneity has not been taken into account in the current modeling because the kinetic theory approach uses a spatial averaging which filters this type of inhomogeneity. In order to take into account this type of inhomogeneity, Brownian dynamics or finer kinetic theory description such as the one proposed by Dhont and Briels (2005) should probably be used. In this paper, the orientation distribution is assumed to depend on the orientation and population conformational coordinates only, reducing $\psi(\mathbf{x}, t, \boldsymbol{\rho}, n)$ to $\psi(\boldsymbol{\rho}, n) . \psi(\boldsymbol{\rho}, n)$ describes the fraction of CNTs oriented in the direction $\boldsymbol{\rho}$ and belonged to a population $n$. It contains information about CNT orientation and aggregation state and the remaining task is to modify the FP equation accordingly.

For an arbitrary population $n$ (where $n \neq 0$ nor 1 ), the population can increase as a result of the aggregation of smaller aggregates $(r<n)$ or the disaggregation of larger aggregates $(r>n)$. On the other hand, the population $n$ can decrease because of the disaggregation of population $n$ forming less entangled aggregates $(r<n)$ or the aggregation of $n$ forming more entangled aggregates $(r>n)$. If a constant aggregation velocity $\left(v_{c}\right)$ and a constant disaggregation velocity of $\left(v_{d}\right)$ are assumed, the following balance expression can be written for the population $n$ :

$$
\begin{aligned}
\left.\frac{\partial \psi(\boldsymbol{\rho}, n)}{\partial t}\right|_{\text {Aggregation }} d n d \boldsymbol{\rho} d t= & \left\{v_{c} \int_{r=0}^{r=n} \psi(\boldsymbol{\rho}, r) d r+v_{d} \int_{r=n}^{r=1} \psi(\boldsymbol{\rho}, r) d r-v_{d} \psi(\boldsymbol{\rho}, n)\right. \\
& \left.\times \int_{r=0}^{r=n} d r-v_{c} \psi(\boldsymbol{\rho}, n) \int_{r=n}^{r=1} d r\right\} d n d \boldsymbol{\rho} d t .
\end{aligned}
$$

The balance equation considering orientation only is given as

$$
\left.\frac{\partial \psi(\boldsymbol{\rho}, n)}{\partial t}\right|_{\text {Orientation }} d n d \boldsymbol{\rho} d t=\left\{-\frac{\partial}{\partial \boldsymbol{\rho}} \cdot\left\{\psi(\boldsymbol{\rho}, n) \frac{d \boldsymbol{\rho}}{d t}\right\}+\frac{\partial}{\partial \boldsymbol{\rho}} \cdot\left\{D_{r}(n) \frac{\partial \psi(\boldsymbol{\rho}, n)}{\partial \boldsymbol{\rho}}\right\}\right\} d n d \boldsymbol{\rho} d t
$$

The FP equation which includes both CNT orientation and aggregation/disaggregation kinetics becomes 


$$
\begin{aligned}
\frac{\partial \psi(\boldsymbol{\rho}, n)}{\partial t}= & \left.\left.\frac{\partial \psi(\boldsymbol{\rho}, n)}{\partial t}\right|_{\text {Orientation }}+\left.\frac{\partial \psi(\boldsymbol{\rho}, n)}{\partial t}\right|_{\text {Aggregation }}\right\} \\
= & \left\{-\frac{\partial}{\partial \boldsymbol{\rho}} \cdot\left\{\psi(\boldsymbol{\rho}, n) \frac{\partial \boldsymbol{\rho}}{\partial t}\right\}+\frac{\partial}{\partial \boldsymbol{\rho}} \cdot\left\{D_{r}(n) \frac{\partial \psi(\boldsymbol{\rho}, n)}{\partial t}\right\}\right\} \\
& +\left\{v_{c} \int_{r=0}^{r=n} \psi(\boldsymbol{\rho}, r) d r+v_{d} \int_{r=n}^{r=1} \psi(\boldsymbol{\rho}, r) d r-v_{d} \psi(\boldsymbol{\rho}, n) \int_{r=0}^{r=n} d r\right. \\
& \left.-v_{c} \psi(\boldsymbol{\rho}, n) \int_{r=n}^{r=1} d r\right\} .
\end{aligned}
$$

For a homogenous flow at steady state

$$
\begin{gathered}
\left\{-\frac{\partial}{\partial \boldsymbol{\rho}} \cdot\left\{\psi(\boldsymbol{\rho}, n) \frac{d \boldsymbol{\rho}}{d t}\right\}+\frac{\partial}{\partial \boldsymbol{\rho}} \cdot\left\{D_{r}(n) \frac{\partial \psi(\boldsymbol{\rho}, n)}{\partial \boldsymbol{\rho}}\right\}\right\}+\left\{v_{c} \int_{r=0}^{r=n} \psi(\boldsymbol{\rho}, r) d r+v_{d} \int_{r=n}^{r=1} \psi(\boldsymbol{\rho}, r) d r\right. \\
\left.-v_{d} \psi(\boldsymbol{\rho}, n) \int_{r=0}^{r=n} d r-v_{c} \psi(\boldsymbol{\rho}, n) \int_{r=n}^{r=1} d r\right\}=0
\end{gathered}
$$

The solution of Eq. (16) should verify the normality condition $\int_{S(0,1)} \int_{0}^{1} \psi(\boldsymbol{\rho}, n) d n d \boldsymbol{\rho}$ $=1$, where $S(0,1)$ represents the unit sphere surface centered at the origin of the orientation conformation space.

The distribution function $\psi(\boldsymbol{\rho}, n)$ can be obtained by solving Eq. (16) and the fourthorder orientation tensor can be expressed as

$$
\mathbf{a}_{4}(n)=\int \boldsymbol{\rho} \otimes \boldsymbol{\rho} \otimes \boldsymbol{\rho} \otimes \boldsymbol{\rho} \psi(\boldsymbol{\rho}, n) d \boldsymbol{\rho} .
$$

The stress contribution due to the presence of CNTs for a multipopulation system can be obtained by generalizing Eq. (3),

$$
\boldsymbol{\tau}=2 \eta \int_{0}^{1} N_{p}(n)\left(\mathbf{a}_{4}(n): \mathbf{D}\right) d n .
$$

The rheological model described above provides a first approximation for describing a suspension with aggregating CNTs and contains four parameters: the concentration and aspect-ratio parameter $\left(N_{p}\right)$, the rotary diffusion coefficient $\left(D_{r}\right)$, and the aggregation and disaggregation velocities $\left(v_{c}\right.$ and $\left.v_{d}\right)$. To simplify the analysis and minimize the degrees of freedom, the following assumptions are made, but other more complex models could be proposed:

i $\quad N_{p}$ varies linearly with the population variable $n$. If we assume that $N_{p}^{\min } \ll N_{p}^{\max }$ and that $N_{p}^{\min } \approx 0$, then $N_{p}(n) \approx N_{p}^{\max } n$ [see Eq. (19a)]. [Note: $N_{p}^{\min }=N_{p}(n=0)$ and $\left.N_{p}^{\max }=N_{p}(n=1)\right]$.

ii In the modeling of associative polymers, a number of authors [Vaccaro and Marrucci (2000); Hernandez Cifre et al. (2003)] assumed that chains within an aggregate network can undergo affine deformation and therefore $D_{r}(n=1) \approx 0$. In the case of CNT suspensions, there is, however, experimental evidence showing that the opposite is true. CNT aggregates tended to rotate in simple shear flow and CNTs within aggregate were observed to be more isotropically orientated compared with disentangled CNTs [Hobbie and Fry (2006); Ma et al. (2007)]. In this 
paper, the rotary diffusion coefficient is therefore assumed to increase linearly with the population $n$ as given in Eq. (19b).

iii $\quad v_{d}$ increases linearly with the shear rate and it takes the form as given in Eq. (19c) where $\dot{\gamma}_{\max }$ is a characteristic shear rate above which the suspension viscosity coincides with the matrix viscosity. At high shear rates, $v_{d}$ remains constant and $v_{d}=v_{d}^{\max }$ [Eq. (19d)].

iv $\quad v_{c}$ decreases linearly with the shear rate and it takes the form as given in Eq. (19e) and for a shear rate higher than $\dot{\gamma}_{\max }$, the aggregation velocity becomes zero [Eq. (19f)]

$$
\begin{aligned}
& N_{p}(n)=N_{p}^{\max } n+N_{p}^{\min }(1-n) \approx N_{p}^{\max } n, \\
& D_{r}(n)=D_{r}^{\max } n, \\
& v_{d}(\dot{\gamma})=\left\{\begin{array}{cl}
v_{d}^{\max }\left(\frac{\dot{\gamma}}{\dot{\gamma}_{\max }}\right) & \text { if } \dot{\gamma} \leqslant \dot{\gamma}_{\max } \\
v_{d}^{\max } & \text { if } \dot{\gamma}>\dot{\gamma}_{\max },
\end{array}\right. \\
& v_{c}(\dot{\gamma})=\left\{\begin{array}{cl}
v_{c}^{\max }\left(\frac{\dot{\gamma}_{\max }-\dot{\gamma}}{\dot{\gamma}_{\max }}\right) & \text { if } \dot{\gamma} \leqslant \dot{\gamma}_{\max } \\
0 & \text { if } \dot{\gamma}>\dot{\gamma}_{\max } .
\end{array}\right.
\end{aligned}
$$

In the AO model, the evolution of CNT aggregates depends on the values of $v_{d}^{\max }$ and $v_{c}^{\max }$ and it would therefore be useful to develop a deeper understanding on how these values affect the population distribution of CNT aggregates.

Integrating Eq. (16) over the unit sphere surface $S(0,1)$ and using the condition that

$$
\int_{S(0,1)}\left\{-\frac{\partial}{\partial \boldsymbol{\rho}} \cdot\left\{\psi(\boldsymbol{\rho}, n) \frac{d \boldsymbol{\rho}}{d t}\right\}+\frac{\partial}{\partial \boldsymbol{\rho}} \cdot\left\{D_{r}(n) \frac{\partial \psi(\boldsymbol{\rho}, n)}{\partial \boldsymbol{\rho}}\right\}\right\} d \boldsymbol{\rho}=0,
$$

the following equation can be derived:

$$
\left\{v_{c} \int_{r=0}^{r=n} C(r) d r+v_{d} \int_{r=n}^{r=1} C(r) d r-v_{d} C(n) \int_{r=0}^{r=n} d r-v_{c} C(n) \int_{r=n}^{r=1} d r\right\}=0,
$$

where $C(n)=\int_{S(0,1)} \psi(\boldsymbol{\rho}, n) d \boldsymbol{\rho}$ and $\int_{0}^{1} C(n) d n=1$.

The population distribution $C(n)$ can be computed from Eq. (21) without considering the orientation distribution. If the values of $v_{d}^{\max }$ and $v_{c}^{\max }$ are fixed, the population distribution $C(n)$, or in a discrete form $C_{i}=C\left(n=n_{i}\right)$, would then be given by Eq. (21). The above analysis shows that the population distribution $C(n)$ only depends on the ratio between $v_{d}^{\max }$ and $v_{c}^{\max }$ (i.e., $\beta=v_{d}^{\max } / v_{c}^{\max }$ ).

\section{Numerical details}

The modified FP equation involved two variables " $\boldsymbol{\rho}$ " and " $n$." The first variable $\boldsymbol{\rho}$ is defined as the surface of the 3D spherical orientation space which has been discretised into $M$ nodes. The second variable $n$ is defined over the one-dimensional (1D) interval [0, 1] with $N$ nodes. The operator involving orientation was discretised using twodimensional triangular linear finite elements with nodes belonging to the surface of the 3D spherical orientation spaces as shown in Fig. 8. The operator involving the second variable $n$ was discretized using $1 \mathrm{D}$ linear finite elements. The size of the orientation 
TABLE I. Typical CPU times required for directly solving the AO model if the model parameters are known. If the model parameters are not known, a longer computation time would be needed to identify the best fit values for fitting the experimental data.

\begin{tabular}{cc}
\hline \hline$M \otimes N$ & CPU time (s) \\
\hline $820 \otimes 20$ & 7 \\
$2100 \otimes 20$ & 26 \\
$820 \otimes 10$ & 2 \\
$820 \otimes 30$ & 17 \\
\hline \hline
\end{tabular}

operator is $(M, M)$ and that of the aggregation operator is $(N, N)$. The global Eq. (16) involves the two variables in a coupled way and therefore the global operator is built on the tensorial product of the two operators and has a size of $(M . M, N . N)$. Table I gives an idea of typical CPU times required for solving the AO model numerically if the model parameters are known. Clearly, if the values of the model parameters are not known, it would take a longer computation time to identify the best fit values for describing the experimental data.

\section{B. Application of the AO model}

The AO model was tested against the experimental evolution of apparent steady shear viscosity for four different concentrations of untreated CNT suspensions $(\phi=0.025 \%$, $0.05 \%, 0.1 \%$, and $0.25 \%$ ) and the fitting results are shown in Fig. 5. Reasonable fittings were obtained and the solutions of the AO model were found to be essentially indepen-
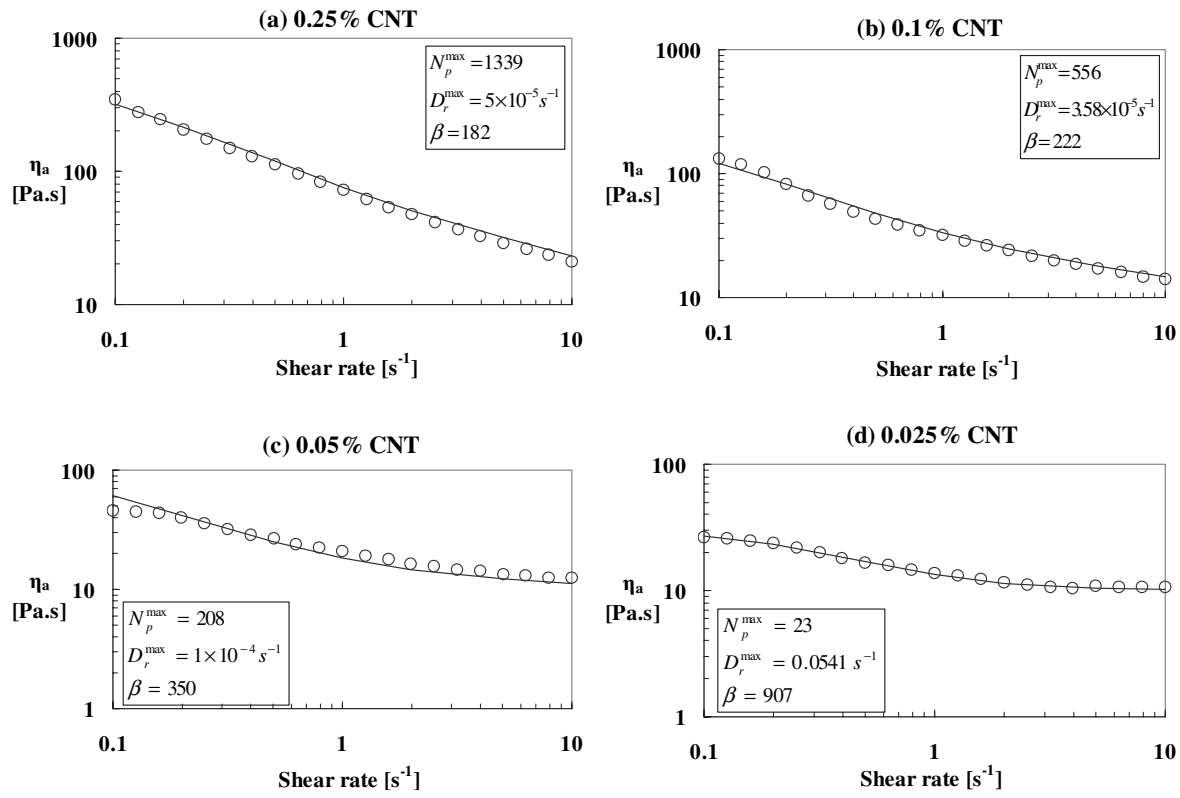

FIG. 5. AO model fitting to experimental $\eta_{a}$ data at different CNT concentration levels: (a) $0.25 \%$, (b) $0.1 \%$, (c) $0.05 \%$, and (d) $0.025 \%$. Experimental data are represented by unfilled circles (O) and the best fit values of $N_{p}^{\max }, D_{r}^{\max }$, and $\beta=v_{d}^{\max } / v_{c}^{\max }$ were obtained from least square fitting. [ $N_{p}^{\min }$ and $\dot{\gamma}_{\max }$ in Eqs. (19a)-(19f) were set to 0 and $100 \mathrm{~s}^{-1}$, respectively]. 


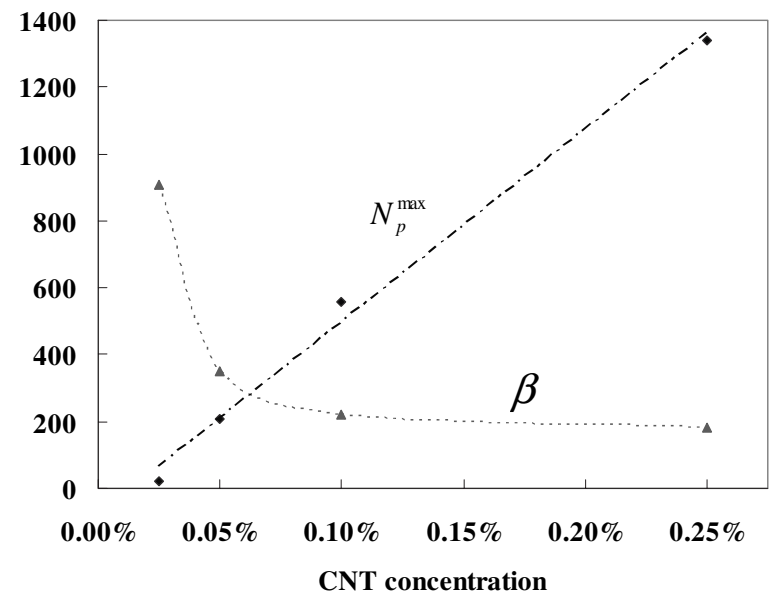

FIG. 6. The best fit values of $N_{p}^{\max }$ and $\beta$ as a function of CNT concentration.

dent of the number of discrete populations $(N)$ used (for $N>10$ ), further confirming the consistency of the model and the convergence of the numerical scheme.

There are three independent adjustable parameters in the AO model fittings and they are $N_{p}^{\max }, D_{r}^{\max }$, and $\beta=v_{d}^{\max } / v_{c}^{\max }$. Least square method was used in the fitting process and the best fit values of parameters $N_{p}^{\max }$ and $D_{r}^{\max }$ were plotted against the CNT concentration as shown in Fig. 6. It has been found that the concentration parameter $N_{p}^{\max }$ scaled linearly with the CNT concentration whereas $\beta$ decreased with increasing concentrations. $\beta$ represents the relative rate of disaggregation to aggregation and the trend in Fig. 7 implies that it is more difficult to disentangle CNTs at high CNT loadings. Intuitively this can be explained by the fact that the inter-spacing between CNTs decreases as the CNT concentration increases and there is a higher chance for the interlocking of CNTs to occur when subject to shear. Finally, the rotary diffusion coefficient parameter, $D_{r}^{\max }$, was found to be in the order of $10^{-4}-10^{-5} \mathrm{~s}^{-1}$ for concentration levels at $0.05 \%$, $0.1 \%$, and $0.25 \%$ whereas the best fit $D_{r}^{\max }$ value for the $0.025 \%$ CNT suspension was identified to be $0.05 \mathrm{~s}^{-1}$.

Figure 7 shows the aggregate population distribution for four different CNT concentrations and two shear rates. The population distribution, $C(n)$, depends on the CNT concentration as well as the shear rate applied. In terms of the effect of shear rate, the simulation results showed that low shear favoured the formation of CNT aggregates with $n$ closes to 1 [Figs. 7(a), 7(c), 7(e), and 7(g)] whereas high shear led to the disaggregation of CNT aggregates and the population distribution skewed towards population $n=0$ in Figs. 7(b), 7(d), 7(f), and 7(h). These simulations results are consistent with the experimental observation that low shear induced aggregation, but the aggregates broke down at high shear, forming smaller aggregates and CNTs with less entanglement. In addition to shear rate, different concentrations of CNT suspensions showed different tendencies to aggregate. $C(n)$ skewed more towards $n=1$ (CNT network) with increasing concentrations, implying suspensions with higher CNT concentration have a higher tendency to aggregate. It was verified that the area under the curve for Figs. 7(a)-7(h) was very close to 1 and the condition $\int_{0}^{1} C(n) d n=1$ was satisfied.

Figure 8 shows the detailed orientation distribution for two CNT populations within a $0.025 \%$ CNT suspension sheared at two different shear rates $\left(0.1\right.$ and $\left.10 \mathrm{~s}^{-1}\right)$. Flow is in the $X$ direction as indicated in Fig. 8(a) and the shear gradient is on the $X-Z$ plane. The 

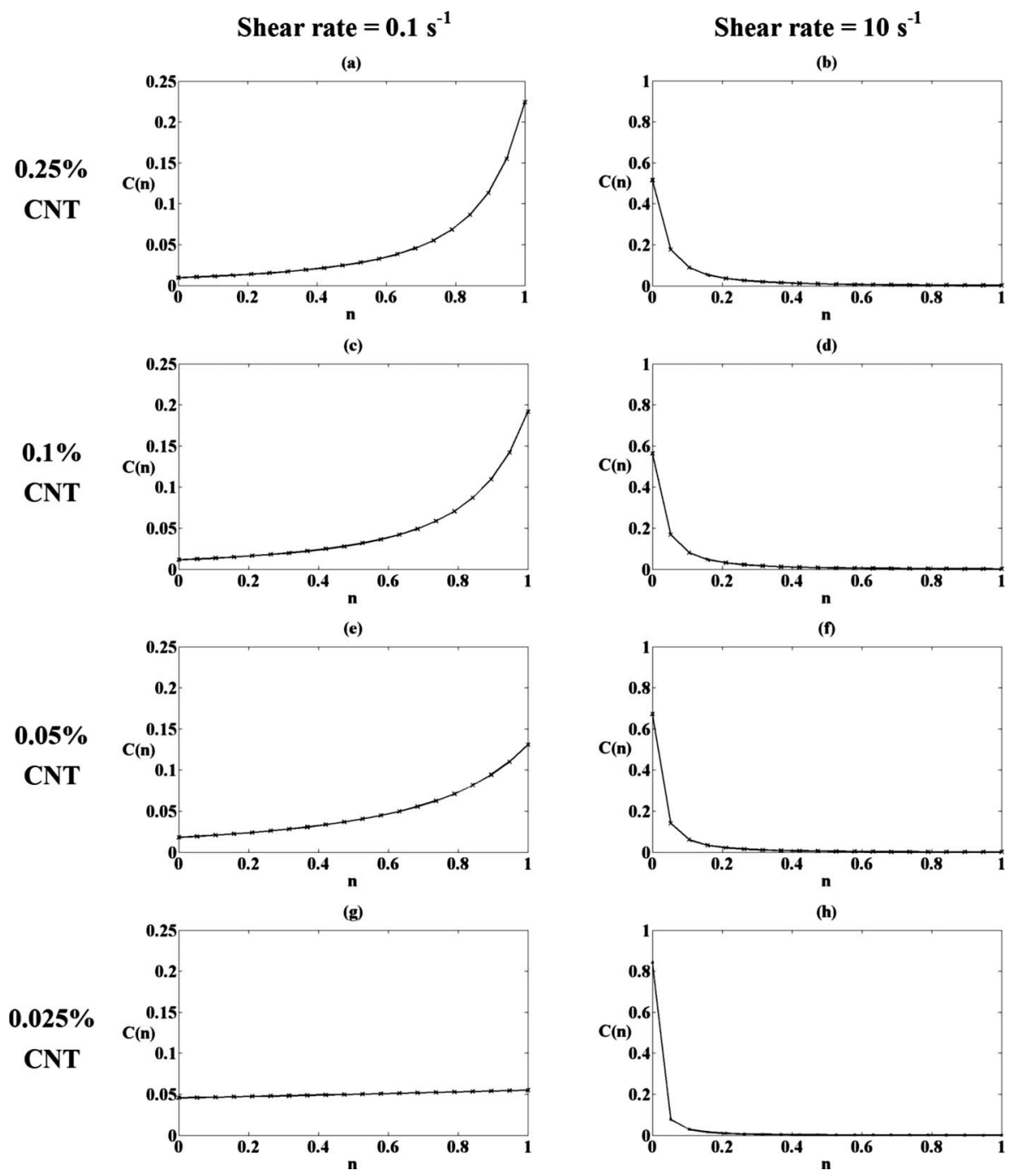

FIG. 7. AO model: The effect of CNT concentration and magnitude of shear rate on the CNT population distributions $C(n)$. This figure shows that CNT tended to aggregate (population distribution skews towards $n$ $=1)$ at high concentration and low shear conditions.

grey scale represents the magnitude of orientation distribution function for free tubes [Figs. 8(a) and 8(c)] and highly entangled CNTs [Figs. 8(b) and 8(d)]. The darker the color, the higher the probability of finding a CNT with an orientation defined by the center point of the unit sphere $(0,0,0)$ and a point on the unit sphere surface. Simulation results showed that in the presence of shear, CNTs tended to orient towards the flow direction $(X)$. It is also clear by comparing Fig. 8(a) to Fig. 8(c) that the degree of alignment in the flow direction increased as the magnitude of shear rate increased and similar results were obtained for CNT aggregate with $n=1$ [see Figs. 8(b) and 8(d)]. Fry et al. (2005) reported the anisotropy of CNTs using the nematic order parameter. The nematic order parameter, or $S(n)$, is a parameter varying from 0 to 1 and the larger the magnitude, the higher the degree of alignment. $S(n)$ associated with the distribution shown in Figs. 8(a) and 8(b) was calculated to be about 0.2 whereas that for Figs. 8(c) 
Free CNTs with $n=0$

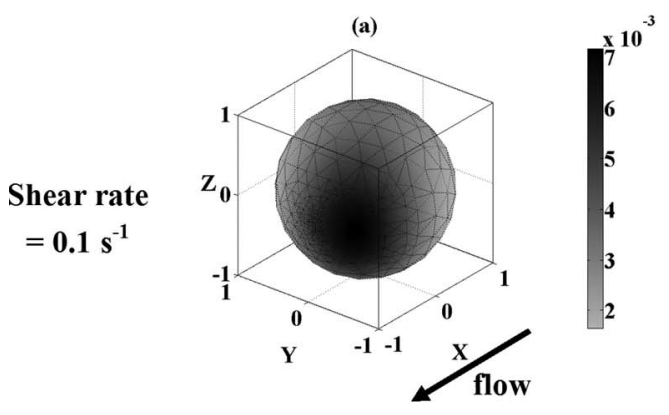

(c)

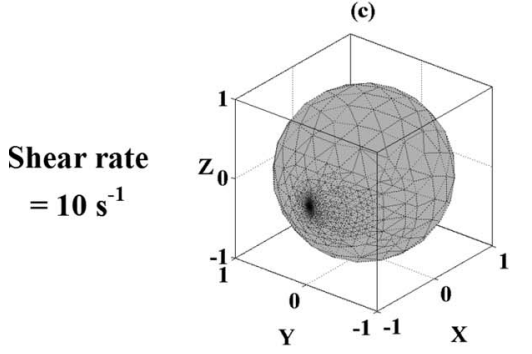

CNT aggregates with $n=1$
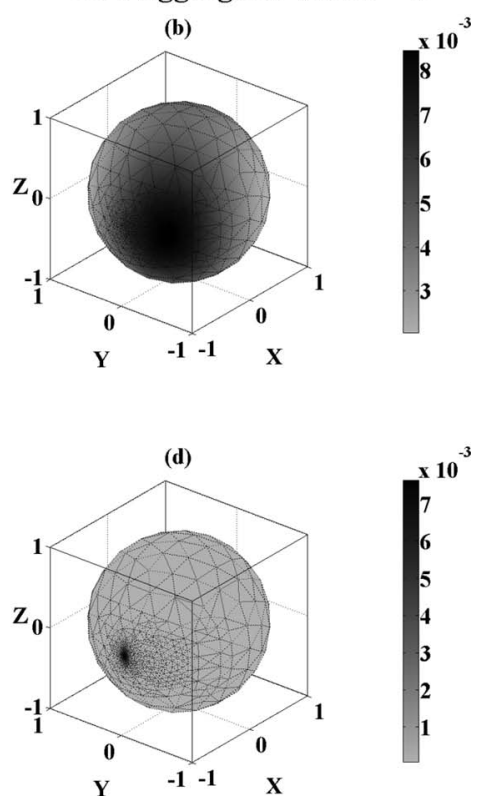

FIG. 8. AO model: Detailed orientation distribution for two CNT populations in a $0.025 \%$ for CNT suspension sheared at $0.1 \mathrm{~s}^{-1}\left[(\mathrm{a})\right.$ and (b)] and at $10 \mathrm{~s}^{-1}[(\mathrm{c})$ and (d)]. Flow is in the $X$ direction as indicated in a and the shear gradient is on the $X-Z$ plane. The color scale represents the magnitude of orientation distribution function for free tubes [(a) and (c)] and highly entangled CNTs [(b) and (d)]. The darker the color, the higher the probability of finding a CNT having an orientation defined by the center point of the unit sphere $(0,0,0)$ and the point on the sphere.

and 8 (d) was close to 0.9 , indicating a higher anisotropy at high shear. $S(n)$ was found to decrease slightly with the population as the rotary diffusion coefficient increases, but this dependence was found to be relatively weak compared with the shear rate effect.

Finally, Fig. 9 shows that the AO model can be used to model the transient viscosity change for a series of step changes in shear rate. The suspension concentration is $0.1 \%$ and the parameters used in the transient modeling were determined from the best fits to steady shear data $\left(N_{p}^{\max }=556, D_{r}^{\max }=3.58 \times 10^{-5}, \beta=222\right)$. The transient shear viscosity data in Fig. 9 were plotted on a linear scale. Although the AO model predicted a small overshoot which was not observed experimentally, there was a reasonable agreement between experimental data and model prediction. The model as presented here does not include LVE and it is anticipated that LVE effects could influence the transient response. Both untreated and treated CNT suspensions do exhibit LVE as reported, for example, by Song and Youn (2005) for untreated and Fan and Advani (2007) for treated. The authors of this paper have been able to include LVE effects into the rheological modeling of treated CNT suspensions [Ma et al. (submitted)]. For completeness, experimental LVE data for the untreated CNT aggregate suspensions are included in the Figs. 10-12 but the inclusion of VE into the AO modeling has proved to be more challenging and will therefore be reported as a separate publication.

\section{DISCUSSION AND CONCLUSIONS}

Untreated CNTs were initially modeled as rigid rods that can rotate and diffuse in a shear flow. It was assumed in the initial modeling that hydrodynamic forces aligned the 


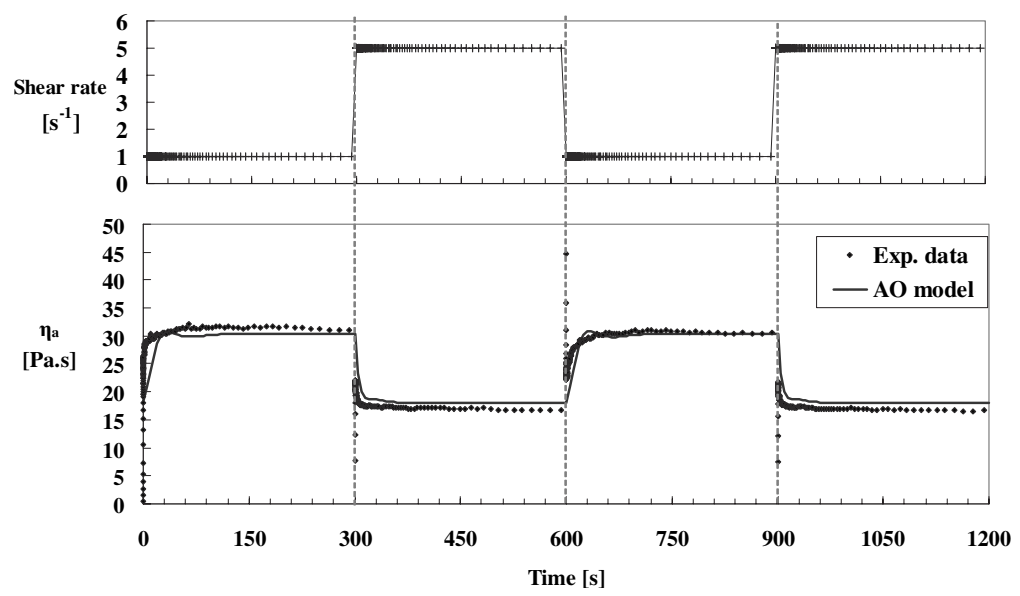

FIG. 9. Time evolution of shear viscosity when the $0.1 \%$ CNT suspension was subjected to a series of step changes in shear rate. The line represents the AO model prediction with parameter values determined from best fits to steady shear data $\left(N_{p}^{\max }=556, D_{r}^{\max }=3.58 \times 10^{-5}, \beta=222\right)$. Transient experimental data are represented by $(\diamond)$.

CNTs in the shear direction, but there were also randomizing events such as Brownian motion and tube-tube interactions that counteracted the hydrodynamic forces in aligning the CNTs. The evolution of CNT orientation was described by a FP equation with a certain rotary diffusion coefficient $\left(D_{r}\right)$ and viscosity contribution due to the presence of CNTs depended on the CNT orientation. In the case of untreated CNT suspensions, initial FP orientation modeling with two adjustable parameters $\left(D_{r}\right.$ and $\left.N_{p}\right)$ failed to capture the low-shear-viscosity enhancement effect as observed experimentally. Furthermore, optical microstructure studies suggested the presence of CNT aggregates at low and intermediate shear rates, leading to the conjecture that besides CNT orientation, aggregation of CNTs had also influenced the shear viscosity of the system as a whole.

The original FP equation in the orientation model was modified to include the experimentally observed effect of aggregates and one additional parameter was added to describe the aggregation/disaggregation kinetics. An AO model was formulated and the

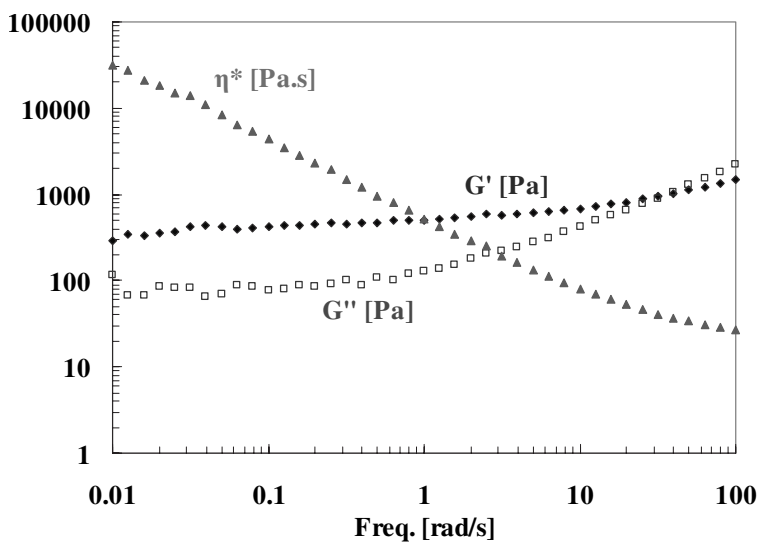

FIG. 10. LVE data $\left(\mathrm{G}^{\prime}\right.$ : storage modulus; $\mathrm{G}^{\prime \prime}$ : loss modulus; $\eta^{*}$ : complex viscosity) for the $0.25 \% \mathrm{CNT}$ suspension ( strain $=1 \%$; temperature $=25^{\circ} \mathrm{C} ; 50 \mathrm{~mm}$ parallel plates). 


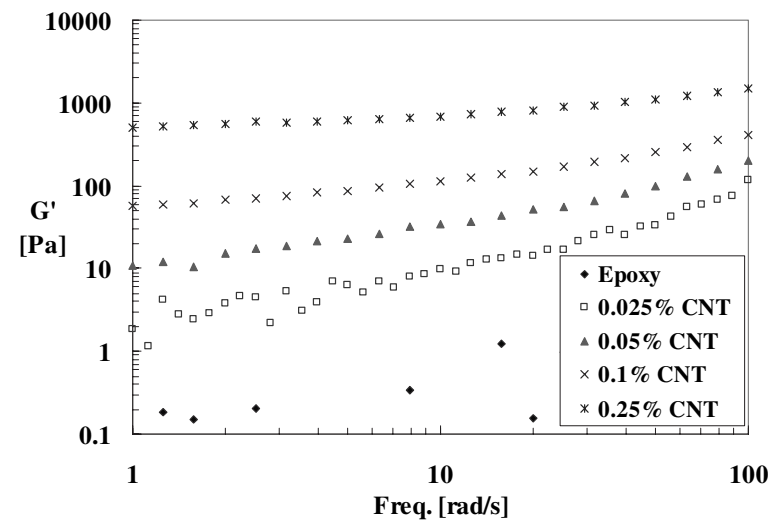

FIG. 11. Dependence of the storage modulus $\left(\mathrm{G}^{\prime}\right)$ on the $\mathrm{CNT}$ concentration (strain $=1 \%$; temperature $=25{ }^{\circ} \mathrm{C} ; 50 \mathrm{~mm}$ parallel plates). The epoxy matrix shows negligible elasticity and the magnitude of $\mathrm{G}^{\prime}$ increases as a function of increasing CNT concentration level.

number of fitting parameters in the model was reduced by introducing a number of simplifying assumptions. A simple version of the AO model contains three fitting parameters $\left(N_{p}^{\max }, D_{r}^{\max }\right.$, and $\left.\beta\right)$ and it was capable of describing the experimental shearthinning characteristic of aggregating CNT suspensions. Based on the best fits to experimental data, the concentration parameter $N_{p}^{\max }$ was found to scale linearly with the actual CNT concentration whereas $\beta$ decreased (asymptotically) with increasing concentration. Since $\beta$ represents the relative rate of disaggregation to aggregation, this simulation results imply that the higher the CNT concentration, the higher the tendency for the suspension to aggregate. The model developed might not be a unique model in terms of describing shear thinning, but it does provide some insight into the physics when an aggregating CNT suspension is subjected to steady shear. The model showed that CNTs aggregated at low shear and the level of entanglement decreased as the shear rate increased. This prediction is consistent with the optical observations and offers a plausible explanation to the significant shear-thinning characteristic of aggregating CNT suspen-

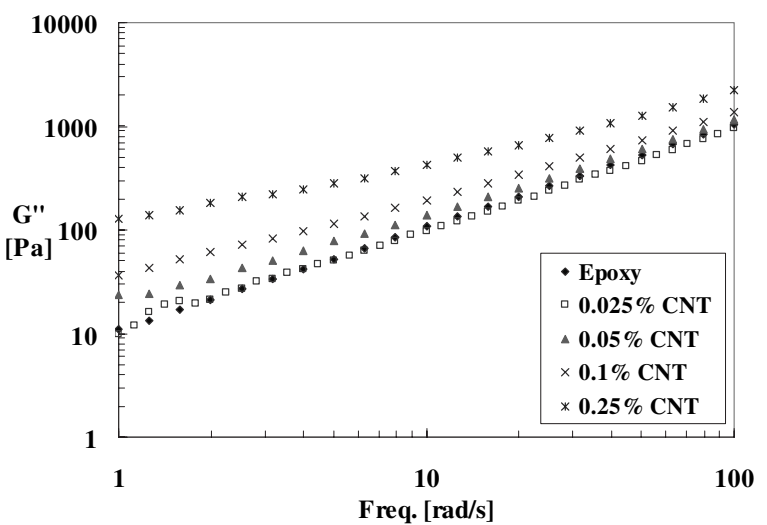

FIG. 12. Dependence of the loss modulus $\left(\mathrm{G}^{\prime \prime}\right)$ on the $\mathrm{CNT}$ concentration (strain $=1 \%$; temperature $=25^{\circ} \mathrm{C}$; $50 \mathrm{~mm}$ parallel plates). $\mathrm{G}^{\prime \prime}$ of the system increases as the CNT concentration increases. 
sions. The AO model contains useful information about CNT orientation and the state of aggregation which is important for controlling or predicting the physical properties of CNT based end-products.

Finally, despite the success of the AO model in describing the steady shear response of aggregating CNT suspensions, the model as currently formulated is essentially only viscous and is therefore not yet capable of predicting the experimentally observed viscoelasticity for CNT aggregate suspensions. Inclusion of viscoelastic modeling into the AO model that also describes the shear-thinning response of CNT aggregate suspensions constitutes an important part of ongoing research and the results will be reported in a future paper.

\section{ACKNOWLEDGMENTS}

The authors would like to thank Professor A. H. Windle and the Department of Materials Science and Metallurgy at the University of Cambridge for providing the base multiwalled carbon nanotubes. A. Ma would also like to thank the Croucher Foundation Scholarship and the Overseas Research Students Awards Scheme (ORSAS) for providing financial support.

\section{References}

Abdel-Goad, M., and P. Pötschke, "Rheological characterization of melt processed polycarbonate-multiwalled carbon nanotube composites," J. Non-Newtonian Fluid Mech. 128, 2-6 (2005).

Advani, S. G., and C. L. Tucker III, "Closure approximations for three-dimensional structure tensors," J. Rheol. 34, 367-386 (1990).

Ammar, A., B. Mokdad, F. Chinesta, and R. Keunings, "A new family of solvers for some classes of multidimensional partial differential equations encountered in kinetic theory modelling of complex fluids," J. Non-Newtonian Fluid Mech. 139, 153-176 (2006).

Batchelor, G. K., "The stress system in a suspension of force-free particles," J. Fluid Mech. 41, 545-570 (1970).

Carvalho, M. S., M. Padmanabhan, and C. W. Macosko, "Single-point correction for parallel disks rheometry," J. Rheol. 38, 1925-1936 (1994).

Chaouche, M., and D. Koch, "Rheology of non-Brownian rigid fiber suspensions with adhesive contacts," J. Rheol. 45, 369-382 (2001).

Chinesta, F., G. Chaidron, and A. Poitou, "On the solution of the Fokker-Planck equation in steady recirculating flows involving short fibre suspensions," J. Non-Newtonian Fluid Mech. 113, 97-125 (2003).

Davis, V. A., L. M. Ericson, A. N. G. Parra-Vasquez, H. Fan, Y. Wang, V. Prieto, J. A. Longoria, S. Ramesh, R. K. Saini, C. Kittrell, W. E. Billups, W. W. Adams, R. H. Hauge, R. E. Smalley, and M. Pasquali, "Phase behavior and rheology of SWNTs in superacids," Macromolecules 37, 154-160 (2004).

Dhont, J. K. G., and W. J. Briels, "Isotropic-nematic spinodal decomposition kinetics," Phys. Rev. E 72 , 031404 (2005).

Du, F., R. C. Scogna, W. Zhou, S. Brand, J. E. Fischer, and K. I. Winey, "Nanotube networks in polymer nanocomposites: Rheology and electrical conductivity," Macromolecules 37, 9048-9055 (2004).

Dupret, F., and V. Verleye, "Modelling the flow of fibre suspensions in narrows gaps," in Advances in the Flow and Rheology of Non-Newtonian Fluids, Rheology Series, edited by D. A. Siginer, D. De Kee, and R. P. Chabra (Elsevier, New York, 1999), pp. 1347-1398.

Fan, Z., and S. G. Advani, "Characterization of orientation state of carbon nanotubes in shear flow," Polymer 46, 5232-5240 (2005).

Fan, Z., and S. G. Advani, "Rheology of multiwall carbon nanotube suspensions," J. Rheol. 51, 585-604 (2007). 
Folgar, F., and C. L. Tucker III, “Orientation behaviour of fibers in concentrated suspensions,” J. Reinf. Plast. Compos. 3, 98-119 (1984).

Fry, D., B. Langhorst, H. Kim, E. Grulke, H. Wang, and E. K. Hobbie, “Anisotropy of sheared carbon-nanotube suspensions," Phys. Rev. Lett. 95, 038304 (2005).

Hand, G. L., "A theory of anisotropic fluids," J. Fluid Mech. 13, 33-46 (1962).

Hernandez Cifre, J. G., Th. M. A. O. M. Barenbrug, J. D. Schieber, and B. H. A. A. van den Brule, "Brownian dynamics simulation of reversible polymer networks under shear using a non-interacting dumbbell model," J. Non-Newtonian Fluid Mech. 113, 73-96 (2003).

Hinch, E. J., and L. G. Leal, "Constitutive equations in suspension mechanics. Part I," J. Fluid Mech. 71, 481-495 (1975).

Hinch, E. J., and L. G. Leal, "Constitutive equations in suspension mechanics. Part II," J. Fluid Mech. 76, 187-208 (1976).

Hobbie, E. K., and D. J. Fry, “Nonequilibrium phase diagram of sticky nanotube suspensions," Phys. Rev. Lett. 97, 036101 (2006).

Hobbie, E. K., and D. J. Fry, "Rheology of concentrated carbon nanotube suspensions," J. Chem. Phys. 126, 124907-1-7 (2007).

Hough, L. A., M. F. Islam, P. A. Janmey, and A. G. Yodh, "Viscoelasticity of single-walled carbon nanotube suspensions," Phys. Rev. Lett. 93, 168102 (2004).

Huang, Y. Y., S. V. Ahir, and E. M. Terentjev, "Dispersion rheology of carbon nanotubes in a polymer matrix," Phys. Rev. B 73, 125422 (2006).

Jeffery, G. B., "The motion of ellipsoidal particles immersed in a viscous fluid ," Proc. R. Soc. London, Ser. A 102, 161-179 (1922).

Keunings, R., "Micro-macro methods for the multiscale simulation viscoelastic flow using molecular models of kinetic theory," in Rheology Reviews, edited by D. M. Binding and K. Walters (British Society of Rheology, London, 2004), pp. 67-98.

Kharchenko, S. B., J. F. Douglas, J. Obrzut, E. A. Grulke, and K. B. Migler, "Flow induced properties of nanotube-filled polymer materials," Nature Mater. 3, 564-568 (2004).

Kim, B., H. Park, and W. M. Sigmund, "Rheological behavior of multiwall carbon nanotubes with polyelectrolyte dispersants," Colloids Surf., A 256, 123-127 (2005).

Kinloch, I. A., S. A. Roberts, and A. H. Windle, "A rheological study of concentrated aqueous nanotube dispersions," Polymer 43, 7483-7491 (2002).

Larson, R. G., The Structure and Rheology of Complex Fluids (Oxford University Press, New York, 1999), pp. 261-355.

Lin-Gibson, S., J. A. Pathak, E. A. Grulke, H. Wang, and E. K. Hobbie, "Elastic flow instability in Nanotube suspensions," Phys. Rev. Lett. 92, 048302 (2004).

Lipscomb, G. G., M. M. Denn, D. H. Hur, and D. V. Boger, "The flow of fiber suspensions in complex geometries," J. Non-Newtonian Fluid Mech. 26, 297-325 (1988).

Ma, A. W. K., F. Chinesta, M. R. Mackley, "The rheology and modelling of chemically treated carbon nanotube suspensions," J. Rheol. (submitted).

Ma, A. W. K., F. Chinesta, T. Tuladhar, and M. R. Mackley, "Filament stretching of Carbon Nanotube suspensions," Rheol. Acta 47, 447-457 (2008a).

Ma, A. W. K., M. R. Mackley, and F. Chinesta, "The microstructure and rheology of carbon nanotube suspensions," Int. J. Mater. Form., 1(2), 75-81 (2008b).

Ma, A. W. K., M. R. Mackley, and S. S. Rahatekar, "Experimental observation on the flow-induced assembly of carbon nanotube suspensions to form helical bands," Rheol. Acta 46, 979-987 (2007).

Macosko, C. W., Rheology: Principles, Measurements and Applications (Wiley/VCH, New York, 1994).

McNally, T., P. Pöschke, P. Halley, M. Murphy, D. Martin, S. E. J. Bell, G. P. Brennan, D. Bein, P. Lemoine, and J. P. Quinn, "Polyethylene multiwalled carbon nanotube composites," Polymer 46, 8222-8232 (2005).

Petrich, M. P., D. L. Koch, and C. Cohen, "An experimental determination of the stress-microstructure relationship in semi-concentrated fiber suspensions," J. Non-Newtonian Fluid Mech. 95, 101-133 (2000).

Petrie, C. J. S., “The rheology of fibre suspensions," J. Non-Newtonian Fluid Mech. 87, 369-402 (1999).

Pötschke, P., T. D. Fornes, and D. R. Paul, "Rheological behavior of multiwalled carbon nanotube / polycar- 
bonate composites," Polymer 43, 3247-3255 (2002).

Rahatekar, S. S., K. K. K. Koziol, S. A. Butler, J. A. Elliott, M. S. P. Shaffer, M. R. Mackley, and A. H. Windle, "Viscosity enhancement of an epoxy resin matrix by addition of multi-wall carbon nanotubes," J. Rheol. 50, 599-610 (2006).

Rincón, E., A. E. Chávez, R. Herrera, and O. Manero, "Rheological modelling of complex fluids: A transient network model with microstates," J. Non-Newtonian Fluid Mech. 131, 64-77 (2005).

Schmid, C. F., and D. J. Klingenberg, "Mechanical flocculation in flowing fiber suspensions," Phys. Rev. Lett. 84, 290-293 (2000).

Schmid, C. F., L. H. Switzer, and D. J. Klingenberg, "Simulations of fiber flocculation: Effects of fiber properties and interfiber friction,” J. Rheol. 44, 781-809 (2000).

Singh, C., M. S. P. Shaffer, and A. H. Windle, "Production of controlled architectures of aligned carbon nanotubes by an injection chemical vapour deposition method," Carbon 41, 359-368 (2003).

Song, Y. S., and J. R. Youn, "Influence of dispersion states of carbon nanotubes on physical properties of epoxy nanocomposites," Carbon 43, 1378-1385 (2005).

Switzer, L. H., and D. J. Klingenberg, "Flocculation in simulations of sheared fiber suspensions," Int. J. Multiphase Flow 30, 67-87 (2004).

Tripathi, A., K. C. Tam, and G. H. McKinley, "Rheology and dynamics of associative polymers in shear and extension: theory and experiments," Macromolecules 39, 1981-1999 (2006).

Vaccaro, A., and G. Marrucci, “A model for the nonlinear rheology of associating polymers," J. Non-Newtonian Fluid Mech. 92, 261-273 (2000).

Xu, J., S. Chatterjee, K. W. Koelling, Y. Wang, and S. E. Bechtel, "Shear and extensional rheology of carbon nanofiber suspensions," Rheol. Acta 44, 537-562 (2005).

Zirnsak, M. A., D. U. Hur, and D. V. Boger, "Normal stresses in fibre suspensions," J. Non-Newtonian Fluid Mech. 54, 153-193 (1994). 